\title{
Taming the Coffee Ring Effect: Enhanced Thermal Control as a Method for Thin-Film Nanopatterning
}

\author{
Rafal Sliz,* Jakub Czajkowski, and Tapio Fabritius
}

Cite This: Langmuir 2020, 36, 9562-9570

Read Online

ABSTRACT: Predicting and controlling a droplet's behavior on surfaces is very complex due to several factors affecting its nature. These factors play a crucial role in colloidal material deposition and related solution-based manufacturing methods such as printing. A better understanding of the processes governing the droplet in the picoliter regime is needed to help develop novel thin-film manufacturing methods and improve the current ones. This study introduces the substrate temperature as a method to control the droplet's behavior during inkjet printing, especially the coffee-ring phenomena, at an unprecedented temperature range $\left(25-250{ }^{\circ} \mathrm{C}\right)$. To explain the particular behavior of the droplet,

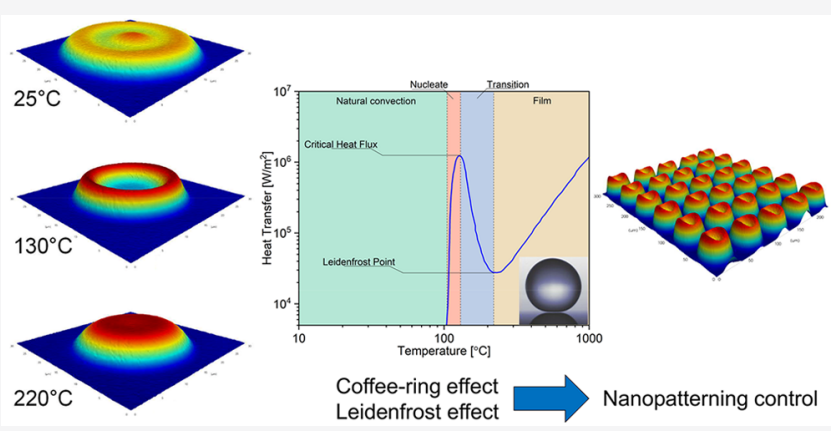
this research associates the creation of specific coffee-ring micro/ nanostructures at elevated temperatures with the Leidenfrost effect that is responsible for creating a vapor pocket under the drying drop. Herein, we combine experimental data and numerical methods to explain the drying dynamic of the picoliter-size droplet on the substrate at elevated temperatures. The achieved results indicate that the coffee-ring effect is correlated with the heat-transfer changes caused by the Leidenfrost effect and can be controlled and used to produce micro/nanostructured thin films without additional processing steps.

\section{INTRODUCTION}

When droplets, containing solid materials or dissolved nonevaporating compounds, dry on a surface, they leave patterns and structures which could be utilized in various applications. ${ }^{1}$ For decades, this phenomenon has been studied to better understand the patterning schemes for coating applications. ${ }^{2-4}$ Recent experimental and theoretical advances have revealed the potential of drop-evaporated patterns in biomedicine and nanotechnology. In biomedical applications, the analysis of the distinctive patterns left from dried drops of biological fluids provides a cost-effective and fast method for detecting and diagnosing certain diseases. ${ }^{5-8}$ From the standpoint of nanotechnology, solution-based processing, material deposition, and the resulting solute patterns have gained significant attention thanks to their applicability to various fields of science and technology. Some of the applications include the manufacturing of conductive thin films of improved transparency, specular holograms, micro- and nanopatterning, and the manufacture of active and passive components. ${ }^{9-14}$ The printing technologies that enable scalable and inexpensive manufacturing of electronic components and devices are particularly promising. ${ }^{15-20}$

Despite the great potential, the prediction and drop control on a solid substrate are very problematic because of the large number of factors governing their natures. The droplet's size, surface tension, liquid/material composition, and viscosity all play roles in its behavior. Furthermore, the substrate and its surface energy, material, pattern, and temperature also affect the drop's behavior in various ways. Finally, the environment (including the surrounding gas, humidity, and temperature) as well as additional stimulants (such as illumination, laser light, and ultrasound vibrations) must be considered. Sefiane, in his review, evaluates the aforementioned factors, their influence on the drop behavior, and the formation of different deposition patterns. ${ }^{21}$ Moreover, there are processes that take place during droplet drying at elevated temperatures, such as Marangoni flows, the coffee-ring effect, and the Leidenfrost effect.

The coffee-ring effect is a pattern left after evaporation by a puddle of liquid containing nonevaporating compounds (Figure 1a). It is mostly induced by two mechanisms: increased evaporation due to the curvature at the drop's edge and the geometric nature of pinning. While pinning restrains the drop from shrinking, a simultaneously increased edge evaporation process induces a flux flow within the droplet. To understand this phenomenon better, several systematic studies have been undertaken. Deegan et al. demonstrated that during drop evaporation a flux of fluid at the edge of the drop leads to the

Received: May 26, 2020

Revised: July 20, 2020

Published: July 22, 2020 


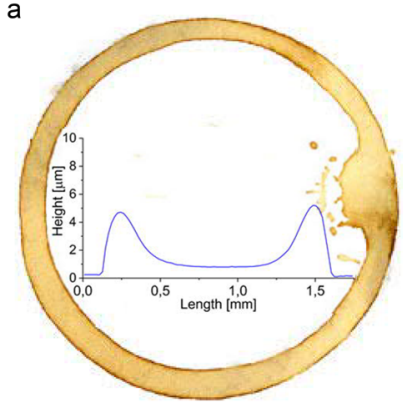

C

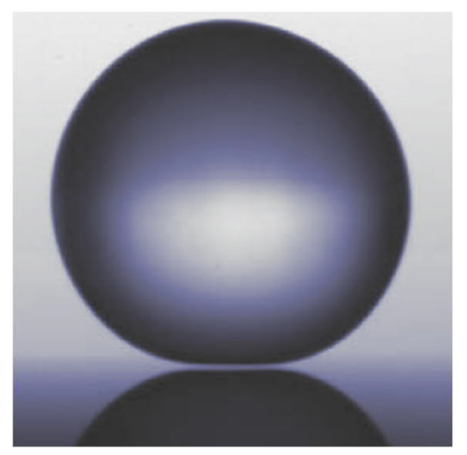

b

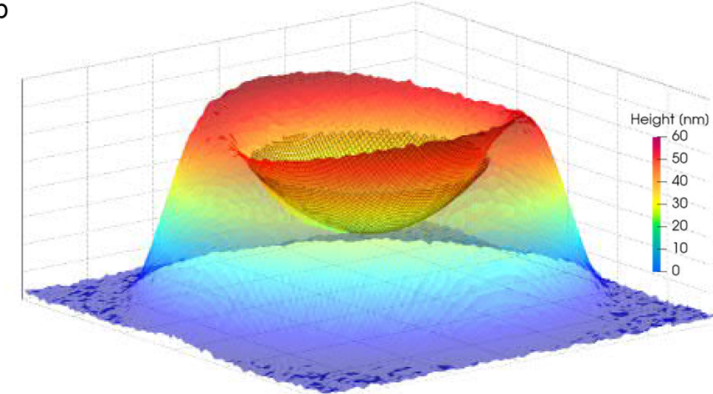

d

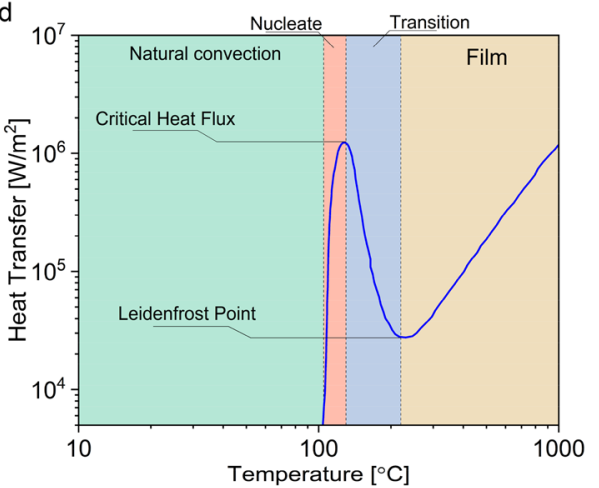

Figure 1. Graphical representation of the coffee-ring and Leidenfrost effects. (a) Coffee-ring pattern made by coffee and its profile after drying. The image and the profilometry results confirm that most of the material remains on the edge of the stain. (b) Three-dimensional fitting of the spherical surface for the concave-plano lens into an inkjet-printed micro/nanostructure that can be used in disposable microscopy diagnostics. ${ }^{30}$ (c) Demonstration of the Leidenfrost effect: the drop of water is hovering over the surface on the vapor cushion. ${ }^{31}$ (d) Leidenfrost heat-transfer curve: the colored areas indicate different regions of drop behavior: natural convection where the drop dries slowly, nucleate boiling area where the heat transfer increases significantly, transition boiling where the increasing temperature reduces the drop drying until reaching the Leidenfrost point, and film boiling where the sustained increase of the substrate temperature results in increased heat transfer and rapid drop evaporation.

growth of solute on the peripheries of the drop. ${ }^{22}$ Yunker et al. emphasized the importance of the suspended particles in the drying droplet due to their interparticle capillary interactions and influence on the coffee-ring effect. ${ }^{23}$ Work conducted by $\mathrm{Hu}$ and Larson describes Marangoni flows, induced by the temperature differences between the contact line and the top of the drop and their role in diminishing the coffee-ring effect. ${ }^{24}$ Similarly, many other groups showed that elevated substrate temperature induces additional heat flux from the surface, leading to increased evaporation of the drop at its edges, significantly affecting the coffee-ring effect. ${ }^{25,26}$ Research conducted by Shen et al. provides the lower limit $\left(D_{c} \gtrsim 10\right.$ $\mu \mathrm{m}$ for $\sim 100 \mathrm{~nm}$ particles) of a droplet's diameter for the successful formation of coffee-ring structures. ${ }^{27}$ Although the coffee ring is often considered to be an undesired phenomenon, reducing the uniformity of the printed layers, when tamed, it can be used advantageously in the manufacturing of patterned thin films (Figure 1b).

The Leidenfrost effect occurs when a liquid drop touches a surface hotter than the liquid's boiling point, creating a vapor layer between the drop and the heated substrate (Figure 1c). ${ }^{28,29}$ The vapor cushion starts forming at temperatures above the socalled critical heat flux, reducing the heat transfer and consequently increasing the droplet drying time (Figure 1d). A progressive increase of the substrate temperature leads to the saturation level where the heat transfer is minimal and the drop drying time is highest: the Leidenfrost point. After this point, increasing the substrate temperature also increases the heat transfer, resulting in shortened drop drying time. The studies and evidence related to the Leidenfrost effect in printing technologies, namely, inkjet printing, are rather limited due to restrictions imposed by the producers of material inkjet printers related to safety considerations at elevated temperatures.

To control the drop behavior on the substrate, researchers proposed a number of methods, including surface treatments (plasma, UV), electrowetting, thermal treatments, solvent surfactants, various particle additives, humidity cycling, and substrate patterning. ${ }^{32-35}$ However, most of the methods require changes in the material composition or deviation from the established protocol, where the functional materials and the solvent composition are carefully adjusted. ${ }^{36-38}$ Altering the developed protocol often undesirably changes the thin-film properties and, consequently, the performance of the final device. Therefore, it is in the interest of the scientific and engineering communities to develop a better understanding of how to precisely control the behavior of the droplet without modifying its material composition or the physiochemical properties of the material beneath. Among the least-invasive methods of controlling the droplet behavior, thermal control of the substrate is considered the most suitable. ${ }^{25,39,40}$ However, many aspects and phenomena remain unanswered, especially for substrate temperatures above $100{ }^{\circ} \mathrm{C}$.

In this work, we focus on the empirical and numerical explanation of drop behavior and various patterns created on the heated substrates by combining the coffee-ring and the Leidenfrost effects to explain the droplet drying process. Importantly, the analysis is performed using materials and conditions relevant to innovative electronic devices (PEDOT:PSS, $\mathrm{ZnO}$, and $\mathrm{Cu}$ nanoparticles) and inkjet printing (picoliter droplet volumes and $25-250{ }^{\circ} \mathrm{C}$ substrate temper- 
a

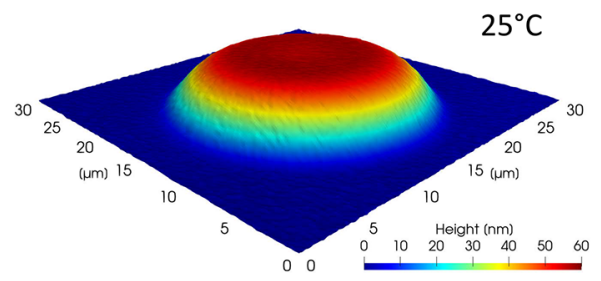

d

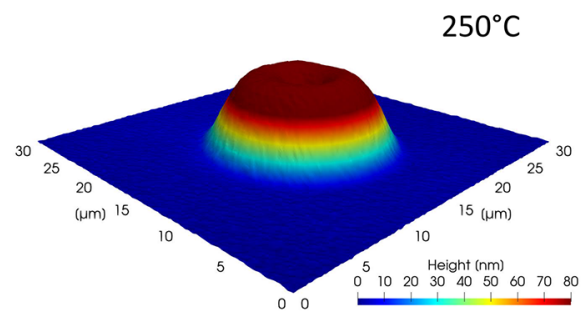

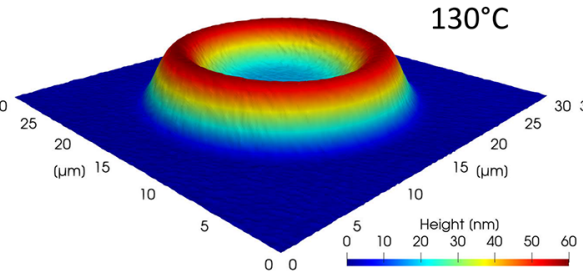

e

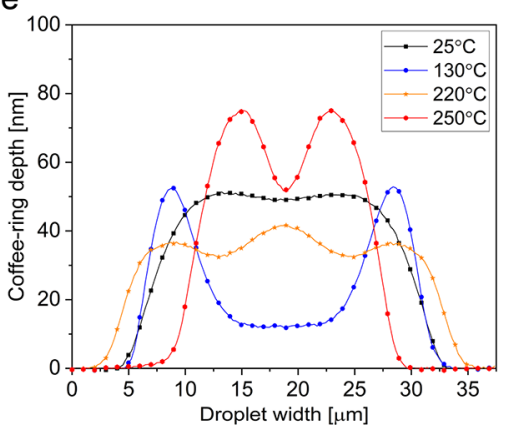

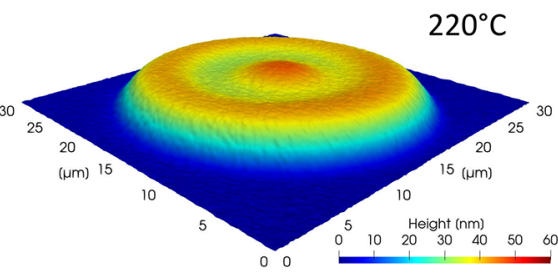

f

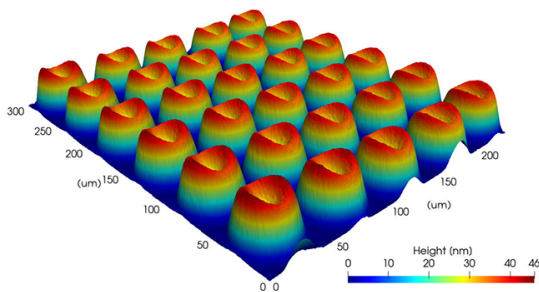

Figure 2. PEDOT:PSS droplet's coffee-ring behavior at various temperatures. The images represent the critical points of the substrate temperature from the perspective of the Leidenfrost effect: (a) Natural convection. (b) Nucleate boiling area where the critical heat flux occurs at $130{ }^{\circ} \mathrm{C}$. (c) Transition boiling where the Leidenfrost effect arises with the Leidenfrost point at $220^{\circ} \mathrm{C}$. (d) Film boiling temperature range where heat transfer increases significantly above $240^{\circ} \mathrm{C}$. (e) Cross-sectional profiles of the droplets at the aforementioned points. The full spectrum of the cross-sectional profiles is demonstrated in Figure S3 in the Supporting Information. (f) Controlled behavior of the droplets of PEDOT:PSS produces a highly ordered nanopattern. The substrate temperature of $120^{\circ} \mathrm{C}$ results in micro/nanostructures of approximately $50 \mu \mathrm{m}$ in diameter and $45 \mathrm{~nm}$ in height. The continuity of the pattern proves that the droplets dry in their impact location. A single nozzle of the Dimatix printer is capable of printing at a speed of 5000 drops/s. Hence, 16 nozzles allow patterning at a speed of approximately $2 \mathrm{~cm}^{2} / \mathrm{s}$.

atures), increasing the impact and applicability of this study. Our printed micro/nanostructures can be utilized to enhance the optical and electrical properties of the optoelectronics devices such as solar cells, detectors, and light-emitting devices. ${ }^{41-43}$

\section{EXPERIMENTAL SECTION}

Printing and Characterization. This section describes in detail the experimental analysis of the drop behavior on the substrate at elevated temperatures for three different inks:

- PEDOT:PSS - PEDOT:PSS 4083 (Heraeus), 2.5 wt \% in $\mathrm{H}_{2} \mathrm{O}$ with the addition of glycerol $1 \mathrm{wt} \%$,

- ZnO IPA - zinc oxide nanoparticles (Sigma-Aldrich 808202, 10 $\mathrm{mL}) 2.5 \mathrm{wt} \%$ in 2-propanol,

- Cu TGME - single-crystal copper nanoparticles (Sigma-Aldrich 907413, $5 \mathrm{~mL}$ ) $2.5 \mathrm{wt} \%$ in triethylene glycol monomethyl ether.

The inks were selected to ensure the usability of the proposed method in the fabrication of optoelectronic devices (PEDOT:PSS, $\mathrm{ZnO}, \mathrm{Cu}$ ) and to better understand the influence of the solvent's boiling point on the droplet behavior (boiling points: IPA, $83^{\circ} \mathrm{C}$; water, 100 ${ }^{\circ} \mathrm{C}$; and TGME, $249^{\circ} \mathrm{C}$ ). The concentration of the solid content was chosen on the basis of our initial tests, which indicated that inks with higher solid content concentrations ( $>4 \mathrm{wt} \%)$ create larger structures but are less prone to creating coffee-ring features. While printouts of inks with lower solid content concentrations (below 2.5 wt \%) demonstrate coffee-ring structures, their height is significantly reduced due to a smaller amount of material. These basic findings are consistent with research showing that the printing of high-concentration inks results in more uniform patterns with reduced or missing coffee-ring structures. $^{44,45}$

The experiment was divided into two parts: the printing process and the achieved micro/nanostructure profile extraction. Inkjet printing was performed with Dimatix material printer DMP-2831 with a $1 \mathrm{pL}$ printing head. As a default, the printer allows the elevation of the substrate temperature up to $60^{\circ} \mathrm{C}$. We upgraded the printer with an additional foil heating system to widen the temperature range from 25 to $350{ }^{\circ} \mathrm{C}$. The temperature was controlled by adjusting the voltage at the contacts of the foil heater beneath the investigated sample. In addition, an implemented thermocouple together with a computercontrolled PID algorithm allowed precise control of the substrate temperature, i.e., compensation for the temperature changes caused by the movements of the printing head.

The main ink used in this research (PEDOT:PSS) was enhanced with the addition of $1 \%$ of glycerol to improve the printing properties and the drop formation. To improve the printing quality, before the printing, the ink was sonicated and degassed. The glass substrate (with a thin $150 \mathrm{~nm}$ layer of conductive ITO) was solvent cleaned in acetone, isopropanol, and methanol. Subsequently, the substrate was placed on the heater and the temperature was adjusted, starting from room temperature $\left(25^{\circ} \mathrm{C}\right)$. Before each print, the substrate was held for $5 \mathrm{~min}$ at a fixed temperature to ensure an even temperature distribution on the substrate surface. The temperature was adjusted in $10^{\circ} \mathrm{C}$ steps from 25 to $250^{\circ} \mathrm{C}$. A printed pattern consisted of a square of printed drops with $40 \mu \mathrm{m}$ spacing. On the basis of the initial printing trials, $40 \mu \mathrm{m}$ spacing was found to be optimal to ensure close packing of the micro/ nanostructures while avoiding drop overlapping. The same steps were applied to print $\mathrm{ZnO}$ IPA and $\mathrm{Cu}$ TGME. Due to the high boiling point of TGME, the range of printing temperatures for $\mathrm{Cu}$ TGME ink was extended to $350^{\circ} \mathrm{C}$.

After the printing process, the substrate was left for eventual drying and transferred for surface characterization, which was performed with an optical profilometer (Bruker Contur GTX-0X) in PSI mode. A surface area of approximately $0.02 \mathrm{~mm}^{2}$ was investigated, and raw data was used for further analysis. The 3D images for PEDOT:PSS and their respective cross-sectional profiles are plotted in Figures 2, S2, and S3. Consequently, dimensional data from the plotted profiles for all inks were extracted and used to analyze the influence of temperature on the drop behavior. To ensure the reliability of the results, for each material and temperature three different droplet profiles were extracted, the depth of the coffee-ring structures was measured, and the standard deviation was calculated (Figure 3). 


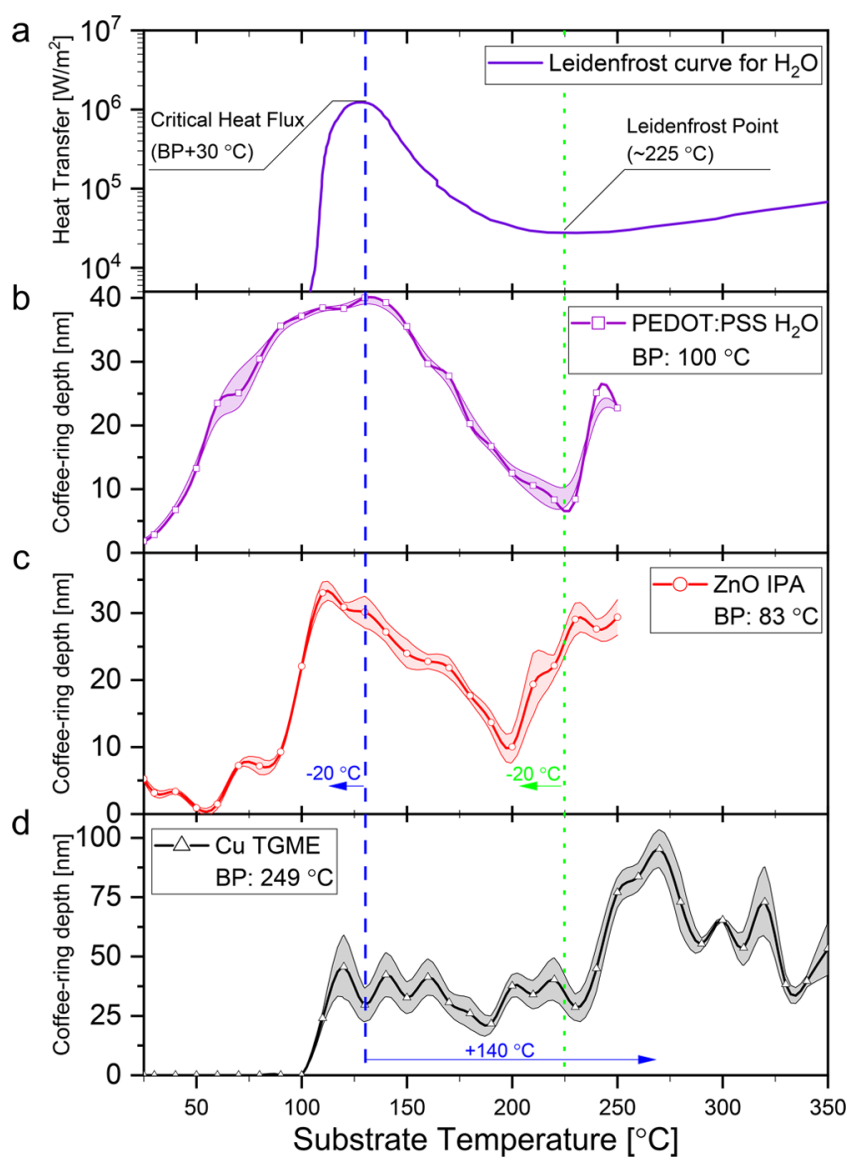

Figure 3. Correlation between the Leidenfrost and coffee-ring effects for various solvents and materials. The standard deviation for each curve is presented as the shadow line. (a) Leidenfrost curve representing the heat transfer between the substrate at an elevated temperature and a droplet of water at the boiling point $(\mathrm{BP})\left(100^{\circ} \mathrm{C}\right)$. The dashed and dotted lines highlight two critical temperature points for water-based inks: the critical heat flux $\left(130^{\circ} \mathrm{C}\right)$ and the Leidenfrost point $\left(220^{\circ} \mathrm{C}\right)$. (b) Change in the depth of the coffee-ring structure for water-based PEDOT:PSS ink in the temperature range of $25-250^{\circ} \mathrm{C}$. (c) Coffee-ring behavior of the droplets at various temperatures for $\mathrm{ZnO}$ IPA ink. The horizontal arrow-lines indicate the shift of the curve (by $20{ }^{\circ} \mathrm{C}$ ) toward lower temperatures caused by the lower boiling point of IPA $\left(83^{\circ} \mathrm{C}\right)$. (d) Coffee-ring behavior of the droplets at various temperatures for $\mathrm{Cu}$ TGME ink. The horizontal arrow indicates the shift of the curve (by approximately $140{ }^{\circ} \mathrm{C}$ ) toward higher temperatures caused by the higher boiling point of TGME $\left(249^{\circ} \mathrm{C}\right)$. The green line shift is not depicted because its location is out of the range of our measurement setup $\left(\sim 379^{\circ} \mathrm{C}\right)$.

Although we attempted to create structures at substrate temperatures of $>250{ }^{\circ} \mathrm{C}$ for water and IPA and $>350{ }^{\circ} \mathrm{C}$ for TGME, the heat flux from the substrate caused inkjet nozzle clogging, resulting in an inability to create a drop. In addition, the use of such high temperatures is questionable due to material degradation.

Modeling. To better understand the phenomena occurring during drop drying on the substrate at elevated temperatures, we performed a set of numerical analyses. The details, critical assumptions, and logic applied are described in the Supporting Information document.

We modeled the drop shape on the heated substrate and the creation of the vapor pocket under the water drop (Figure 4). A MATLAB environment was used to calculate the dimensions and shape of the vapor pocket under the droplet. The achieved ultra-high-resolution profiles of the droplets at various temperatures were transferred to the Autodesk AutoCAD environment for verification and further conversion to the DXF format. Furthermore, we used this format to simulate various aspects of the drop behavior by using the COMSOL Multiphysics software. Our model describes the 2D stationary behavior of the drop surrounded by the ambient atmosphere and the heated substrate at the bottom, for which the size of the vapor pocket is taken into consideration. The achieved results and the drops' altered shapes were used to conduct an analysis of the fluid behavior within the water droplets (Figure 5) and the surrounding environment (Figure S6) at analogous temperatures. To better understand the heat distribution, additional modeling focused on creating an artificial environment where the droplet is surrounded (sides and top) by a perfect heat insulator and the only source of heat transferred to the droplet is the direct one from the substrate through the vapor cushion (Figure S7). Additional plots demonstrating the heat transfer from the bottom of the droplet only and the pressure distribution within the drop (Figure S5) are provided in the Supporting Information.

\section{RESULTS AND DISCUSSION}

Printing and Characterization. Our experimental studies involved testing the drop behavior at the substrate, with temperatures elevated from room temperature $\left(25^{\circ} \mathrm{C}\right)$ to 250 ${ }^{\circ} \mathrm{C}$ for water and IPA and $350{ }^{\circ} \mathrm{C}$ for TGME. The $3 \mathrm{D}$ images in Figure $2 \mathrm{a}-\mathrm{d}$ show the influence of the substrate temperature on the creation of the PEDOT:PSS structures affected by the coffee-ring effect. The selection of depicted temperatures is noncontingent: they represent critical stages relevant to various boiling phases related to the Leidenfrost effect for water. Figure $2 \mathrm{e}$ provides a more precise analysis of the created structures by depicting cross-sectional profiles of the 3D images. Figure $2 \mathrm{f}$ demonstrates an example of a highly ordered pattern created in one processing step where the behavior of the drop is controlled by the substrate temperature. A full data set of the $3 \mathrm{D}$ images in the form of animations and cross-sectional profiles of the structures with a $10{ }^{\circ} \mathrm{C}$ step increase is available in the Supporting Information.

Plots in Figure 3 demonstrate the correlation between the Ledenfrost effect and the creation of various material coffee-ring micro/nanostructures of different shapes. For reference, Figure 3 a shows the Leidenfrost heat-transfer curve for water, signifying two important temperature points (critical heat transfer (130 ${ }^{\circ} \mathrm{C}$ ) and Leidenfrost point $\left.220^{\circ} \mathrm{C}\right)$ ).

The plot for water-based PEDOT:PSS (Figure 3b) demonstrates the depth of the coffee-ring structures for each substrate temperature $\left(25-250^{\circ} \mathrm{C}\right)$ with a resolution of $10^{\circ} \mathrm{C}$. The plot is allocated on the same temperature scale as the Leidenfrost curve to highlight the correlation between the heattransfer changes and the depth of the coffee-ring structures.

Between 25 and $130{ }^{\circ} \mathrm{C}$, the coffee-ring structures deepen as the heat transfer increases. At $130{ }^{\circ} \mathrm{C}$, where the heat transfer reaches its maximum, the depth of the coffee ring is the largest, suggesting that the increasing heat transfer is responsible for increasing the capillary effect and, consequently, the size of the coffee-ring structure. According to the Leidenfrost effect, the continuous temperature rise, until approximately $220^{\circ} \mathrm{C}$, results in decreased heat transfer between the substrate and the droplet due to the formation of a vapor cushion under the drop. Accordingly, our experimental results show that at substrate temperatures $>130^{\circ} \mathrm{C}$, a continuous increase in the temperature diminishes the coffee-ring effect, which is in agreement with the Leidenfrost effect. Consequently, after reaching the second critical point (Leidenfrost point $220{ }^{\circ} \mathrm{C}$ ), elevated heat transfer between the substrate and the droplet can be observed. Our experimental results also agree with this behavior because at approximately $220^{\circ} \mathrm{C}$ the coffee-ring shape reappears. 
a

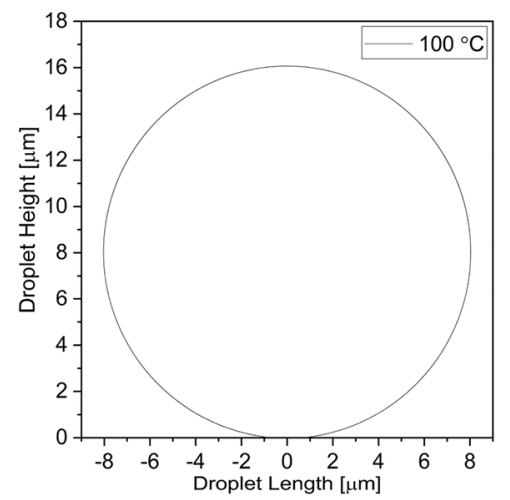

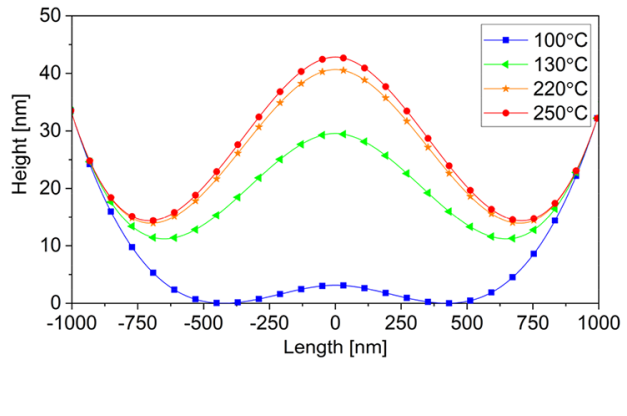

C

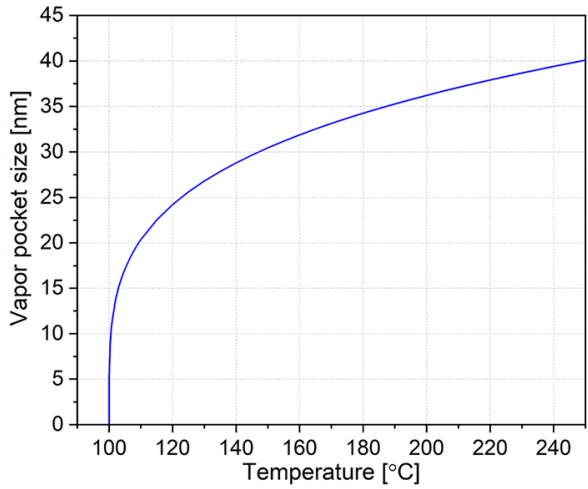

Figure 4. Numerical analysis of the droplet behavior on the substrate at elevated temperature. (a) Simulation of the shape of the $2.17 \mathrm{pL}$ water droplet at the substrate at elevated temperature. (b) Magnified droplet-substrate interface and the shape of the vapor pocket beneath the droplet at various substrate temperatures. The full spectrum of the vapor pocket profiles is demonstrated in Figure S4 in the Supporting Information. (c) Calculated size of the vapor pocket at various temperatures. For temperatures below $100{ }^{\circ} \mathrm{C}$ (water boiling point), the pocket-size equation is unsolvable because of $\Delta T$, which cannot be smaller than or equal to zero. Respective plots for IPA and TGME (Figure S1) are provided in the Supporting Information.

a

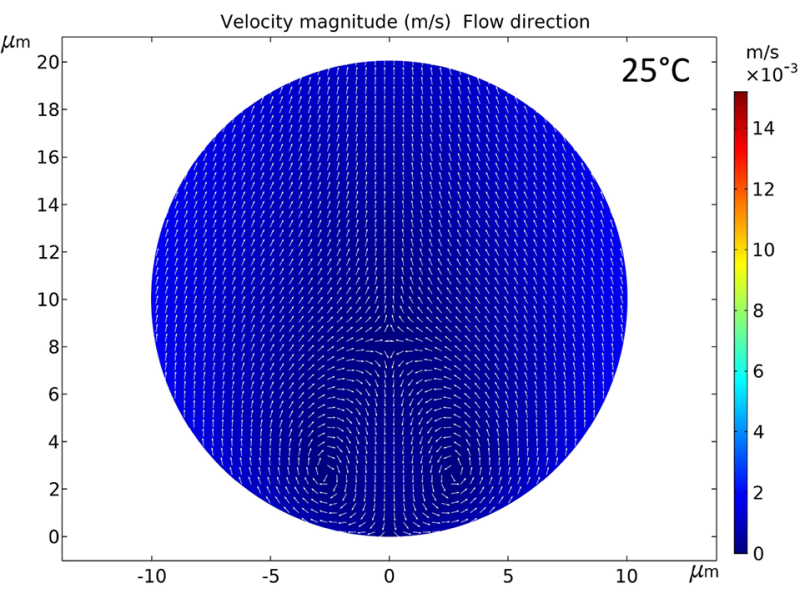

C

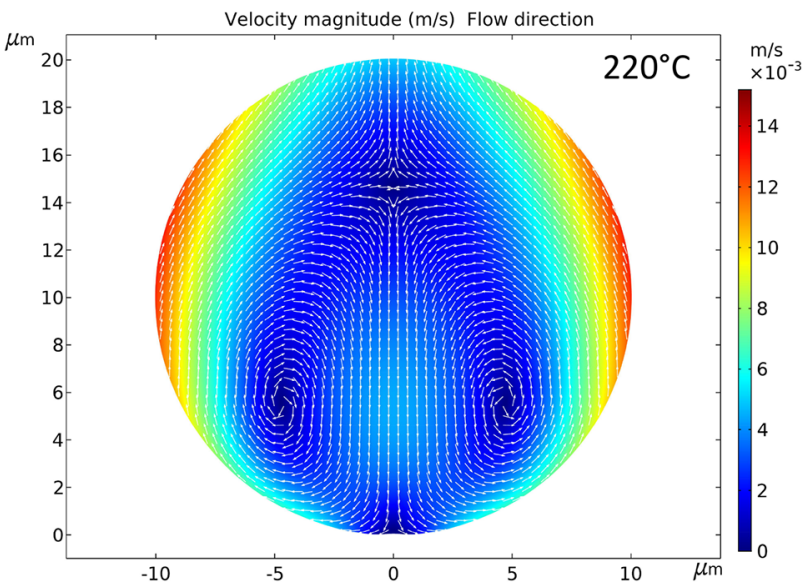

b

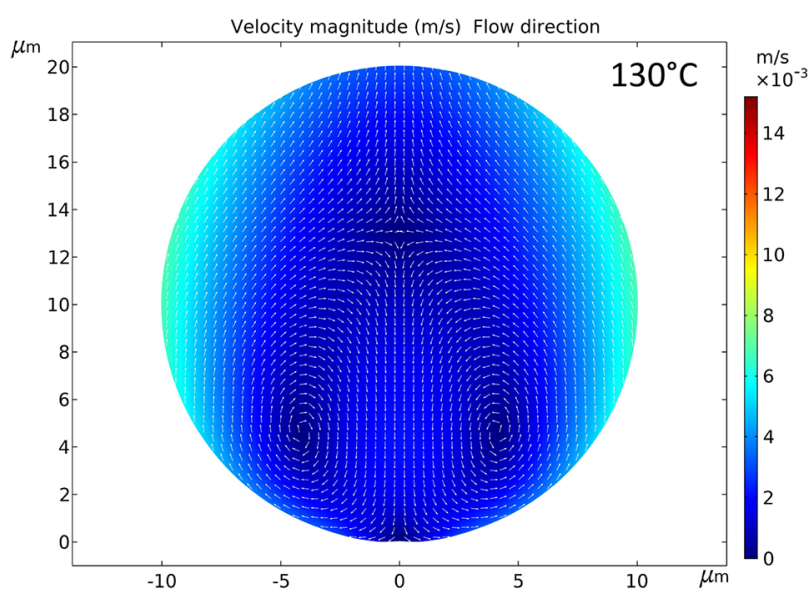

d

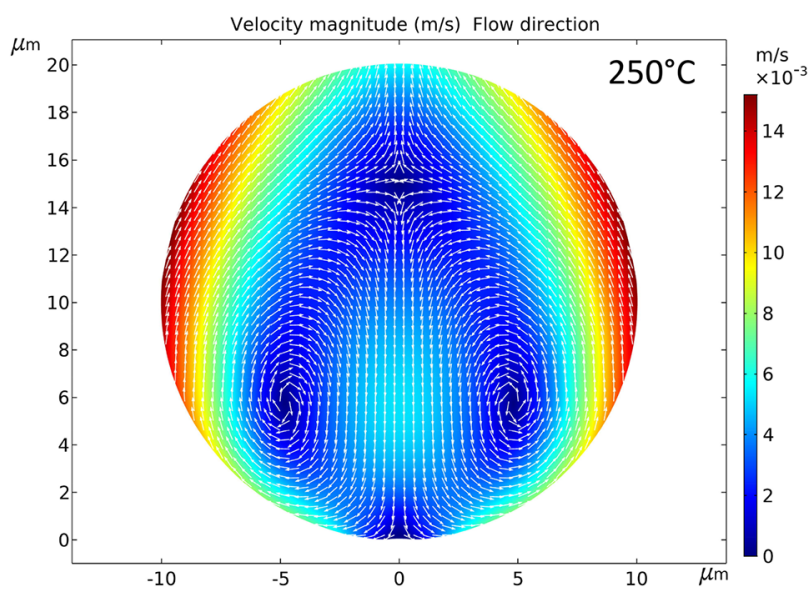

Figure 5. Flow velocity and direction within the water drop at various substrate temperatures. (a) At $25^{\circ} \mathrm{C}$, the flow is almost negligible and the middle vortex is located approximately $6 \mu \mathrm{m}$ from the bottom of the drop while the side vortices are present $2.5 \mu \mathrm{m}$ above the bottom of the drop. With increasing substrate temperature, the flow velocity is increasing, especially at the sides of the droplet. (b) At $130{ }^{\circ} \mathrm{C}$, the velocity of the flow at the sides reaches $6 \times 10^{-3} \mathrm{~m} / \mathrm{s}$. Also, the central vortex is rising significantly while the side vortices move up and aside. For temperatures of (c) 220 and (d) 250 ${ }^{\circ} \mathrm{C}$, the flow velocity reaches $12 \times 10^{-3}$ and $15 \times 10^{-3} \mathrm{~m} / \mathrm{s}$, respectively. In both cases, the central vortex forms approximately $15 \mu \mathrm{m}$ from the bottom of the droplet. The side vortices occur $5.5 \mu \mathrm{m}$ from the bottom of the drop and $5 \mu \mathrm{m}$ to the side of the drop's center.

To verify our experimental results for PEDOT:PSS, we conducted an analogous experiment with different solvents and materials: $\mathrm{ZnO}$ IPA and $\mathrm{Cu}$ TGME. The acquired results presented in Figure $3 \mathrm{c}, \mathrm{d}$ indicate very similar coffee-ring behavior with one crucial difference: the curve is shifted horizontally by approximately $-20{ }^{\circ} \mathrm{C}$ and $+140{ }^{\circ} \mathrm{C}$ for $\mathrm{ZnO}$ IPA and $\mathrm{Cu}$ TGME, respectively. We attributed this shift to the difference in the boiling points for water $\left(100^{\circ} \mathrm{C}\right)$, IPA $\left(83^{\circ} \mathrm{C}\right)$, 
and TGME $\left(249^{\circ} \mathrm{C}\right)$. The analysis of measurement errors in Figure 3 suggests a high consistency of measurements for PEDOT:PSS and ZnO IPA. Meanwhile, the increased standard deviation for the $\mathrm{Cu}$ TGME ink could be correlated with a significantly larger temperature range used in the experiment.

On the basis of the collected data, we derived a simplistic model where the shape of the pattern is related to the energy transfer affected by the Leidenfrost effect. The highest energy transfer occurs at the critical heat flux point, resulting in the largest micro/nanostructures. In contrast, the energy transfer between the substrate and the droplet at the Leidenfrost point is the lowest, resulting in rather uniform structures deprived of the coffee-ring effect. On the basis of the Leidenfrost curve, the assumption that the critical heat flux occurs at a particular temperature (boiling point of the solvent plus $30^{\circ} \mathrm{C}$ ) creates the opportunity to predict the temperatures and relevant heattransfer values for solvents with different boiling points and consequently control the formation of micro/nanostructures regardless of the solvents used.

Modeling. For water at $100{ }^{\circ} \mathrm{C}$, the capillary length equals $2.5 \mathrm{~mm}$, which is about 250 times larger than the radius of the studied inkjet-printed droplets with a volume of $2.17 \mathrm{pL}$. Therefore, we assumed almost perfectly spherical drops as depicted in Figure 4a, and in our considerations we have applied the evaporation models proposed by Biance and Soto. ${ }^{31,46}$ The calculated values were used to plot the drop shape versus substrate temperature, and Figure $4 \mathrm{~b}$ emphasizes the droplet bottom shape change at critical temperatures of 100, 130, 220, and $250{ }^{\circ} \mathrm{C}$. The curve depicted in Figure $4 \mathrm{c}$ demonstrates the changes in the size of the vapor pocket in the full range of applied substrate temperatures. Additional considerations regarding the influences of thermal conductivity, surface tension, and nanoparticles on the droplet behavior for water, IPA, and TGMA are provided in the Supporting Information.

The simulated shapes of the droplets have consequently been used to analyze the flow behavior within the droplets themselves, as plotted in Figure 5. The colors indicate the speed of the flow, while the arrows specify the direction. The velocity of the fluid within the drop, especially the outlying areas, increases with the temperature of the substrate. Interestingly, the flow creates three vortex points that change their location with increasing temperature. Although the vortices are also present in the droplet at lower temperatures, the flow velocity is almost negligible when compared to higher substrate temperatures.

The velocity flow in the droplets' surrounding atmosphere depicted in Figure S6 suggests a significant temperature-induced increase in the flows (internal and external) on the side of the droplet toward the top center. The additional plots that artificially exclude the surrounding heat and focus on the heat transfer in the bottom area of the droplet (Figure S7) suggest that without the heat from the surrounding air the flow velocity within the droplet slows down significantly by an order of magnitude. Therefore, the critical aspect for picoliter droplets is that the source/direction of the heat is changing at higher temperatures.

Our results suggest that there is a correlation between the creation of the coffee-ring effect micro/nanostructures and the heat-transfer changes associated with the Leidenfrost effect. In principle, the larger the heat transfer between the substrate and the droplet, the deeper the coffee-ring structure. However, a few aspects require further analysis and consideration.

First, Celestini et al. predicted that droplets of micrometer size, after impacting the heated surface, will take off due to the breakdown of the lubrication regime. ${ }^{47}$ Bouillant et al. demonstrated that for quasi-spherical droplets the internal flow symmetry is distorted and the droplet starts to roll on the surface. ${ }^{29}$ As seen in Figure $2 \mathrm{f}$, the patterns are undistorted and none of the droplets take off. Importantly, it applies to the whole range of substrate temperatures and materials used in this research. The difference comes from the fact that our droplets consist of solid material. We assume that the material immobilizes the droplet, limiting it to its initial impact location during the lubrication regime by creating a confining perimeter that is pinning the droplet. It allows the creation of the vapor pocket under the droplet but at the same time restricts the horizontal movement of the droplets.

Second, although the correlation between the coffee-ring effect and the heat transfer is visible, the modeling results (Figure 5) indicate that the flow velocity within the droplet increases with the substrate temperature and it is not affected by the heat-transfer variations between the substrate and the droplet induced by the size of the vapor pocket under the droplet.

Additional simulations of the surrounding atmosphere (Figures S6 and S7) demonstrate its critical influence on the droplet drying behavior. Therefore, the critical aspect for picoliter droplets is the change in the heat source/direction at elevated temperatures.

Herein we propose an explanation of the water-based droplet behavior at the critical temperature points. Importantly, for different solvents, the critical temperature points will vary according to their boiling-point temperatures.

At room temperature $\left(25^{\circ} \mathrm{C}\right)$, the droplet falls on the surface and is in contact with the surface. The surface energy of the drop keeps its shape almost perfectly spherical (Figure 4a). The flow in the droplet occurs but its velocity is almost negligible. Slow drying leads to an even distribution of the material.

At lower temperatures $\left(\leq 130{ }^{\circ} \mathrm{C}\right)$ most of the heat is delivered from the substrate to the droplet through direct contact or a very small vapor pocket. When the vapor pocket occurs, it does not significantly affect the heat transfer but together with the created vortices keeps the material from settling in the middle of the drop, resulting in large coffee-ring nano/microstructures.

As the substrate's temperature rises $\left(130-220{ }^{\circ} \mathrm{C}\right)$, the amount of heat transferred from the substrate to the bottom of the droplet is only slightly increasing because of the enlarged vapor pocket that limits the transfer (due to higher temperature and consequently reduced surface tension of the droplet and increased vapor's density and viscosity, according to Soto's equation). At the same time, the substrate more intensively heats the surrounding air and consequently the whole surface of the droplet (Figure S6). According to Baek et al., the high temperature that induces a decrease in viscous stress and an increase in the surface-tension gradient is responsible for the appearance of the central-spot structure and more even material distribution. $^{48}$

When the temperature surpasses the Leidenfrost point $(>220$ ${ }^{\circ} \mathrm{C}$ ), the heat from the top and sides is a dominant because the large vapor pocket keeps the droplet hovering, limiting the heat transfer from the substrate, whereas the surrounding heat causes a rapid evaporation of the drop. Although the elevated temperature also reduces the surface tension of the droplet and increases the vapor density and viscosity, the heat influx from the air significantly reduces the evaporation time. We assume that in this regime the drop is much denser at the 
moment when the material starts to settle on the surface of the substrate.

Our modeling is in agreement with experimental observations conducted by Bhardwaj et al., where two vortices are created at the bottom of the nanoliter droplet. ${ }^{49}$ Importantly, the simulated azimuthal relocation of the flow vortices within the droplets has also been confirmed and experimentally studied by Askounis et al. ${ }^{50}$

The proposed droplet's behavior at various temperatures is also supported by the experimental results of the varying diameter of the coffee-ring structures presented in Figure 2e and Figure S3. Up to $130{ }^{\circ} \mathrm{C}$, the diameter of the coffee-ring structures does not change significantly. For $220^{\circ} \mathrm{C}$, the coffeering structures spread due to the droplet's reduced surface tension and vapor's increased density and viscosity, caused by the elevated temperature. Finally, at very high temperatures $\left(\geq 250{ }^{\circ} \mathrm{C}\right)$, the coffee-ring structures become narrower due to rapid evaporation induced by the surrounding heat. We have considered other factors that might affect the width of the nano/ microstructures, i.e., spreading of the drop upon impacting the substrate. However, for the inks used in this research, the calculated values of the Weber and Reynolds numbers are relatively small, meaning that the spreading of the drop upon impact is negligible because the deformation of the drop is governed by the viscous stress, surface tension, and inertial forces, imposing an almost ideal spherical shape. ${ }^{51}$ It leads us to the assumption that the width of the structure is related to the formation of the vapor pocket and heat-transfer changes occurring during the drying process.

Although our modeling efforts do not fully explain the creation of the nano/microstructures, they emphasize the importance of the surrounding heat on the droplet drying process, which seems to be more crucial than the heat transfer from the bottom and its changes induced by the vapor pocket.

Also, we recognize the deficiency of our stationary model where the simulated values are calculated when the droplet stabilizes on the surface at particular temperatures. However, the following dynamic processes that occur after settling (dynamic changes of the vapor pocket size and shape, vibrations of the droplet, and changes in the droplet radius or its physical parameters during evaporation, to name a few) need further study and more sophisticated models.

Although a wide range of temperatures has been applied to understand the dynamics of the process and to gather data related to the relationship between the coffee-ring effect and the Leidenfrost effect, the full range might not be suitable for all materials and applications. Most of the solution-based electronic hole and electron transport materials (HTL and ETL) require elevated temperature annealing to remove the solvent residues or promote oxidation. ${ }^{52}$ The proposed method makes it possible to combine the annealing and patterning manufacturing steps. For instance, PEDOT:PSS and $\mathrm{ZnO}$ applied in the experiment are usually annealed in the temperature range of $110-130^{\circ} \mathrm{C}$, creating the opportunity to utilize maximum heat transfer to anneal and create the nanopatterns at the same time. Although temperatures $>130{ }^{\circ} \mathrm{C}$ might trigger the degradation of the electrical properties of PEDOT:PSS in which the conductivity of the thin films is declining, ${ }^{53}$ the higher spectrum of temperatures might be suitable for another material that is widely used in optoelectronics, $\mathrm{ZnO}$, especially the sol-gel method where the thin films can be heat treated and patterned at almost the same time. $^{54}$
To improve the control of the droplet behavior further, additional experimental and numerical investigations are needed. More research is needed to better understand how the droplet material penetrates the vapor cushion and settles on the surface while the drop is still hovering and the cooling aspects of the flows within the drop and their influence on the vapor pocket. Although Shen et al. provide information about the influence of humidity on coffee-ring formation, further research is needed to understand the impact of environmental factors better. ${ }^{27}$ Nonetheless, the revealed correlation between the coffee-ring and the Leidenfrost effects in the wide spectrum of substrate temperatures offers the possibility to control the drop behavior for the creation of desired micro/nanostructures.

\section{CONCLUSIONS}

This research provides evidence for the association between the creation of the coffee-ring micro/nanostructures and the heattransfer variations induced by the Leidenfrost effect. The unprecedented temperature range applied in this research reveals the correlation and the opportunity for extended control of the drop behavior by the substrate temperature for various inks. The largest coffee-ring structures can be created by setting the temperature of the substrate to a value where critical heat flux occurs (boiling point of the droplet's solvent plus $30^{\circ} \mathrm{C}$ ). This assumption creates the opportunity to predict the temperatures and relevant heat-transfer trends for solvents with different boiling points and consequently control the formation of micro/nanostructures regardless of the solvents used. The obtained results indicate that the substrate temperature control can be used for the large-scale creation of micro/ nanostructures that can enhance the optical and electrical properties of thin-film devices without introducing additional manufacturing steps.

\section{ASSOCIATED CONTENT}

\section{Supporting Information}

The Supporting Information is available free of charge at https://pubs.acs.org/doi/10.1021/acs.langmuir.0c01560.

Modeling details; modeled change of the vapor pocket for various temperatures and hosting liquids; drop shape over the whole spectrum of temperature with a temperature step of $10{ }^{\circ} \mathrm{C}$; animations that presents the droplet changes in a more comprehensive manner; full set of cross-sectional profiles of the droplets at various temperatures; modeling results of the vapor pocket creation under a $2.17 \mathrm{pL}$ droplet at various substrate temperatures; modeling of the pressure distribution within the drop at the various substrate temperatures; and modeled velocity flow within the droplet and in the surrounding atmosphere under various heat-transfer conditions (PDF)

Animation demonstrating the correlation between the formation of the coffee ring structures and heat transfer changes associated with the Leidenfrost effect (MP4)

\section{AUTHOR INFORMATION}

\section{Corresponding Author}

Rafal Sliz - Optoelectronics and Measurement Techniques Unit, Faculty of Information Technology and Electrical Engineering,

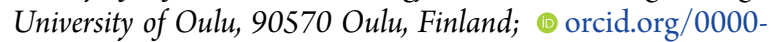
0002-7224-2426; Email: rafal.sliz@oulu.fi 


\section{Authors}

Jakub Czajkowski - Optoelectronics and Measurement Techniques Unit, Faculty of Information Technology and Electrical Engineering, University of Oulu, 90570 Oulu, Finland; Microsoft, HoloLens Optics Finland, 02150 Espoo, Finland

Tapio Fabritius - Optoelectronics and Measurement Techniques Unit, Faculty of Information Technology and Electrical Engineering, University of Oulu, 90570 Oulu, Finland; () orcid.org/0000-0003-4729-8740

Complete contact information is available at: https://pubs.acs.org/10.1021/acs.langmuir.0c01560

\section{Author Contributions}

R.S. conceived the ideas and designed the project, conducted the experiment and made measurements, and wrote the article with contributions from T.F. J.C. conducted numerical analysis and initial image processing. T.F. supported the project and commented on the article.

\section{Notes}

The authors declare no competing financial interest.

\section{ACKNOWLEDGMENTS}

This research was supported by a postdoctoral research fellow grant from the Academy of Finland (grant no. 296890). The authors also express their gratitude for the financial support received from the Academy of Finland's FIRI funding (grant no. 320017). The authors thank Flavia Dinca for her comments and contribution.

\section{REFERENCES}

(1) Deegan, R. D. Pattern Formation in Drying Drops. Phys. Rev. E: Stat. Phys., Plasmas, Fluids, Relat. Interdiscip. Top. 2000, 61, 475-485.

(2) Uno, K.; Hayashi, K.; Hayashi, T.; Ito, K.; Kitano, H. Particle Adsorption in Evaporating Droplets of Polymer Latex Dispersions on Hydrophilic and Hydrophobic Surfaces. Colloid Polym. Sci. 1998, 276, 810-815.

(3) Tay, B. Y.; Edirisinghe, M. J. Time-Dependent Geometrical Changes in a Ceramic Ink Droplet. Proc. R. Soc. London, Ser. A 2002, 458, 2039-2051.

(4) Bonn, D.; Eggers, J.; Indekeu, J.; Meunier, J. Wetting and Spreading. Rev. Mod. Phys. 2009, 81, 739-805.

(5) Sefiane, K. On the Formation of Regular Patterns from Drying Droplets and Their Potential Use for Bio-Medical Applications. J. Bionic Eng. 2010, 7, S82-S93.

(6) Wong, T. S.; Chen, T. H.; Shen, X.; Ho, C. M. Nanochromatography Driven by the Coffee Ring Effect. Anal. Chem. 2011, 83, 1871-1873.

(7) Yakhno, T. A.; Kazakov, V. V.; Sanina, O. A.; Sanin, A. G.; Yakhno, V. G. Drops of Biological Fluids Drying on a Hard Substrate: Variation of the Morphology, Weight, Temperature, and Mechanical Properties. Tech. Phys. 2010, 55, 929-935.

(8) Gorr, H. M.; Zueger, J. M.; McAdams, D. R.; Barnard, J. A. SaltInduced Pattern Formation in Evaporating Droplets of Lysozyme Solutions. Colloids Surf., B 2013, 103, 59-66.

(9) Layani, M.; Gruchko, M.; Milo, O.; Balberg, I.; Azulay, D.; Magdassi, S. Transparent Conductive Coatings by Printing Coffee Ring Arrays Obtained at Room Temperature. ACS Nano 2009, 3, 35373542.

(10) Slabov, V.; Vinogradov, A. V.; Yakovlev, A. V. Inkjet Printing of Specular Holograms Based on a Coffee-Ring Effect Concave Structure. J. Mater. Chem. C 2018, 6, 5269-5277.

(11) Redinger, D.; Molesa, S.; Yin, S.; Farschi, R.; Subramanian, V. An Ink-Jet-Deposited Passive Component Process for RFID. IEEE Trans. Electron Devices 2004, 51, 1978-1983.
(12) Dan, B.; Wingfield, T. B.; Evans, J. S.; Mirri, F.; Pint, C. L.; Pasquali, M.; Smalyukh, I. I. Templating of Self-Alignment Patterns of Anisotropic Gold Nanoparticles on Ordered SWNT Macrostructures. ACS Appl. Mater. Interfaces 2011, 3, 3718-3724.

(13) Secor, E. B.; Prabhumirashi, P. L.; Puntambekar, K.; Geier, M. L.; Hersam, M. C. Inkjet Printing of High Conductivity, Flexible Graphene Patterns. J. Phys. Chem. Lett. 2013, 4, 1347-1351.

(14) Green, A. A.; Hersam, M. C. Colored Semitransparent Conductive Coatings Consisting of Monodisperse Metallic SingleWalled Carbon Nanotubes. Nano Lett. 2008, 8, 1417-1422.

(15) Singh, M.; Haverinen, H. M.; Dhagat, P.; Jabbour, G. E. Inkjet Printing-Process and Its Applications. Adv. Mater. 2010, 22, 673-685.

(16) Kopola, P.; Aernouts, T.; Sliz, R.; Guillerez, S.; Ylikunnari, M.; Cheyns, D.; Välimäki, M.; Tuomikoski, M.; Hast, J.; Jabbour, G.; Myllylä, R.; Maaninen, A. Gravure Printed Flexible Organic Photovoltaic Modules. Sol. Energy Mater. Sol. Cells 2011, 95, 1344-1347.

(17) Hyun, W. J.; Secor, E. B.; Hersam, M. C.; Frisbie, C. D.; Francis, L. F. High-Resolution Patterning of Graphene by Screen Printing with a Silicon Stencil for Highly Flexible Printed Electronics. Adv. Mater. 2015, 27 (1), 109-115.

(18) Hu, G.; Kang, J.; Ng, L. W. T.; Zhu, X.; Howe, R. C. T.; Jones, C. G.; Hersam, M. C.; Hasan, T. Functional Inks and Printing of TwoDimensional Materials. Chem. Soc. Rev. 2018, 47, 3265-3300.

(19) Haverinen, H. M.; Myllylä, R. A.; Jabbour, G. E. Inkjet Printing of Light Emitting Quantum Dots. Appl. Phys. Lett. 2009, 94, 073108.

(20) Choi, H. W.; Zhou, T.; Singh, M.; Jabbour, G. E. Recent Developments and Directions in Printed Nanomaterials. Nanoscale 2015, 7, 3338-3355.

(21) Sefiane, K. Patterns from Drying Drops. Adv. Colloid Interface Sci. 2014, 206, 372-381.

(22) Deegan, R. D.; Bakajin, O.; Dupont, T. F.; Huber, G.; Nagel, S. R.; Witten, T. A. Contact Line Deposits in an Evaporating Drop. Phys. Rev. E: Stat. Phys., Plasmas, Fluids, Relat. Interdiscip. Top. 2000, 62, 756765

(23) Yunker, P. J.; Still, T.; Lohr, M. A.; Yodh, A. G. Suppression of the Coffee-Ring Effect by Shape-Dependent Capillary Interactions. Nature 2011, 476, 308-311.

(24) Hu, H.; Larson, R. G. Marangoni Effect Reverses Coffee-Ring Depositions. J. Phys. Chem. B 2006, 110, 7090-7094.

(25) Soltman, D.; Subramanian, V. Inkjet-Printed Line Morphologies and Temperature Control of the Coffee Ring Effect. Langmuir 2008, 24, 2224-2231.

(26) Parsa, M.; Harmand, S.; Sefiane, K.; Bigerelle, M.; Deltombe, R. Effect of Substrate Temperature on Pattern Formation of Nanoparticles from Volatile Drops. Langmuir 2015, 31, 3354-3367.

(27) Shen, X.; Ho, C. M.; Wong, T. S. Minimal Size of Coffee Ring Structure. J. Phys. Chem. B 2010, 114, 5269-5274.

(28) Quéré, D. Leidenfrost Dynamics. Annu. Rev. Fluid Mech. 2013, $45,197-215$.

(29) Bouillant, A.; Mouterde, T.; Bourrianne, P.; Lagarde, A.; Clanet, C.; Quéré, D. Leidenfrost Wheels. Nat. Phys. 2018, 14, 1188-1192.

(30) Vilmi, P.; Varjo, S.; Sliz, R.; Hannuksela, J.; Fabritius, T. Disposable Optics for Microscopy Diagnostics. Sci. Rep. 2015, 5, $2-7$.

(31) Soto, D. Non-Wetting Drops: From Impacts to Self-Propulsion; Université Pierre et Marie Curie, 2014.

(32) Nguyen, T. A. H.; Biggs, S. R.; Nguyen, A. V. Manipulating Colloidal Residue Deposit from Drying Droplets: Air/Liquid Interface Capture Competes with Coffee-Ring Effect. Chem. Eng. Sci. 2017, 167, $78-87$.

(33) Park, J.; Moon, J. Control of Colloidal Particle Deposit Patterns within Picoliter Droplets Ejected by Ink-Jet Printing. Langmuir 2006, $22,3506-3513$.

(34) Sliz, R.; Suzuki, Y.; Nathan, A.; Myllyla, R.; Jabbour, G. Organic Solvent Wetting Properties of UV and Plasma Treated ZnO Nanorods: Printed Electronics Approach. Proc. SPIE 2012, 8477, 84771G.

(35) Mampallil, D.; Eral, H. B. A Review on Suppression and Utilization of the Coffee-Ring Effect. Adv. Colloid Interface Sci. 2018, $252,38-54$. 
(36) Sliz, R.; Lejay, M.; Fan, J. Z.; Choi, M.-J.; Kinge, S.; Hoogland, S.; Fabritius, T.; García de Arquer, F. P.; Sargent, E. H. Stable Colloidal Quantum Dot Inks Enable Inkjet-Printed High-Sensitivity Infrared Photodetectors. ACS Nano 2019, 13, 11988-11995.

(37) Wang, F.; Cao, Y.; Chen, C.; Chen, Q.; Wu, X.; Li, X.; Qin, T.; Huang, W. Materials toward the Upscaling of Perovskite Solar Cells: Progress, Challenges, and Strategies. Adv. Funct. Mater. 2018, 28, 1803753.

(38) Williams, S. T.; Rajagopal, A.; Chueh, C. C.; Jen, A. K. Y. Current Challenges and Prospective Research for Upscaling Hybrid Perovskite Photovoltaics. J. Phys. Chem. Lett. 2016, 7, 811-819.

(39) Kim, J. H.; Park, S. B.; Kim, J. H.; Zin, W. C. Polymer Transports inside Evaporating Water Droplets at Various Substrate Temperatures. J. Phys. Chem. C 2011, 115, 15375-15383.

(40) Sliz, R.; Suzuki, Y.; Fabritius, T.; Myllyla, R. Influence of Temperature on Wetting Properties of Thin Films in Organic Solar Cells Applications. Colloids Surf., A 2014, 443, 182-187.

(41) He, X.; Gao, F.; Tu, G.; Hasko, D.; Hüttner, S.; Steiner, U.; Greenham, N. C.; Friend, R. H.; Huck, W. T. S. Formation of Nanopatterned Polymer Blends in Photovoltaic Devices. Nano Lett. 2010, 10, 1302-1307.

(42) Menard, E.; Meitl, M. A.; Sun, Y.; Park, J. U.; Shir, D. J. L.; Nam, Y. S.; Jeon, S.; Rogers, J. A. Micro- and Nanopatterning Techniques for Organic Electronic and Optoelectronic Systems. Chem. Rev. 2007, 107, $1117-1160$

(43) Nie, Z.; Kumacheva, E. Patterning Surfaces with Functional Polymers. Nat. Mater. 2008, 7, 277-290.

(44) Brutin, D. Influence of Relative Humidity and Nano-Particle Concentration on Pattern Formation and Evaporation Rate of Pinned Drying Drops of Nanofluids. Colloids Surf., A 2013, 429, 112-120.

(45) Lee, H. H.; Fu, S. C.; Tso, C. Y.; Chao, C. Y. H. Study of Residue Patterns of Aqueous Nanofluid Droplets with Different Particle Sizes and Concentrations on Different Substrates. Int. J. Heat Mass Transfer 2017, 105, 230-236.

(46) Biance, A. L.; Clanet, C.; Quéré, D. Leidenfrost Drops. Phys. Fluids 2003, 15, 1632-1637.

(47) Celestini, F.; Frisch, T.; Pomeau, Y. Take off of Small Leidenfrost Droplets. Phys. Rev. Lett. 2012, 109, 1-5.

(48) Baek, J. M.; Yi, C.; Rhee, J. Y. Central Spot Formed in Dried Coffee-Water-Mixture Droplets: Inverse Coffee-Ring Effect. Curr. Appl. Phys. 2018, 18, 477-483.

(49) Bhardwaj, R.; Fang, X.; Attinger, D. Pattern Formation during the Evaporation of a Colloidal Nanoliter Drop: A Numerical and Experimental Study. New J. Phys. 2009, 11, No. 075020.

(50) Askounis, A.; Kita, Y.; Kohno, M.; Takata, Y.; Koutsos, V. Influence of Local Heating on Marangoni Flows and Evaporation Kinetics of Pure Water Drops. Langmuir 2017, 33, 5666-5674.

(51) Castanet, G.; Caballina, O.; Lemoine, F. Drop Spreading at the Impact in the Leidenfrost Boiling. Phys. Fluids 2015, 27, No. 063302.

(52) Raoufi, D. Synthesis and Microstructural Properties of $\mathrm{ZnO}$ Nanoparticles Prepared by Precipitation Method. Renewable Energy 2013, 50, 932-937.

(53) Vitoratos, E.; Sakkopoulos, S.; Dalas, E.; Paliatsas, N.; Karageorgopoulos, D.; Petraki, F.; Kennou, S.; Choulis, S. A. Thermal Degradation Mechanisms of PEDOT:PSS. Org. Electron. 2009, 10, 6166.

(54) Znaidi, L. Sol-Gel-Deposited ZnO Thin Films: A Review. Mater. Sci. Eng., B 2010, 174, 18-30. 